\section{ADVANTAGES OF THE SEGMENTAL NONDIVIDED INTERCOSTAL MUSCLE FLAP To the Editor:}

The intercostal muscle has been increasingly used to protect and revascularize bronchial stumps and anastomoses after pneumonectomy, sleeve pneumonectomy, lung transplantation, and bronchial sleeve resection. ${ }^{1}$ The muscle can be mobilized with a generous flap of parietal pleura to increase coverage. This flap allows protection of the stump or anastomosis, early revascularization of the bronchus, ${ }^{1}$ and prevention of bronchopleural fistula in case of dehiscence. The flap can be easily prepared during thoracotomy, before spreading the ribs, and left posteriorly in the costovertebral groove protected with a wet gauze during the lung procedure. If not used, the flap can be placed again between the ribs while closing the thoracotomy. However, sometimes the need for this flap cannot be anticipated preoperatively (thus, if required, it has not been prepared). Alternatively, a flap prepared while opening the chest, as decided at preoperative workup, would not be required because of intraoperative strategy changes (eg, a simple lobectomy instead of a sleeve resection or pneumonectomy, or an exploratory thoracotomy because of unexpected metastatic disease or locally advanced disease). In these situations, the surgeon would add operative time in preparing the unnecessary flap or have

\footnotetext{
The Editor welcomes submissions for possible publication in the Letters to the Editor section that consist of commentary on an article published in the Journal or other relevant issues. Authors should: • Include no more than 500 words of text, three authors, and five references. - Type with double-spacing. - See http://jics.ctsnetjournals.org/misc/ifora.shtml for detailed submission instructions. - Submit the letter electronically via jtcvs.editorialmanager. com. Letters commenting on an article published in the JTCVS will be considered if they are received within 6 weeks of the time the article was published. Authors of the article being commented on will be given an opportunity of offer a timely response ( 2 weeks) to the letter. Authors of letters will be notified that the letter has been received. Unpublished letters cannot be returned.
}

a useless intercostal muscle deeply crushed by the retractor at the thoracotomy site. The latter situation requires mobilization of other flaps.

The segmental mobilization of an intercostal muscle flap has been described and repeatedly advocated to decrease postoperative pain in patients undergoing thoracotomy. ${ }^{2,3}$ This technique avoids crushing the intercostal neurovascular bundle during rib spreading with the retractor. Mobilization from the rib is required only at the level where the retractor is placed and takes only a few minutes, much less than the full isolation of the muscle. At the end of the procedure, the ribs are approximated in the usual fashion according to the surgeon's preference.

This easy technique is extremely useful to reduce postoperative pain and to make the intercostal flap available in case of unexpected and complicated surgical procedures. Also, if the flap is not required as anticipated at preoperative workup, the minimal segmental mobilization allows easy closure of the chest without further maneuvers and avoids having a "foreign body" in the posterior aspect of the chest during the lung procedure. This technique is now routinely used at our center, speeding chest opening and allowing bronchial protection when required.

\section{Federico Venuta, $M D^{a}$ Marco Anile, $M D^{a}$}

Erino A. Rendina, $M D^{b}$

${ }^{a}$ Cattedra di Chirurgia Toracica Policlinico Umberto I

Dipartimento Paride Stefanini Università di Roma SAPIENZA V.le del Policlinico Rome, Italy

${ }^{b}$ Ospedale S Andrea

Department of Thoracic Surgery

Università di Roma SAPIENZA

Rome, Italy

\section{References}

1. Rendina EA, Venuta F, Ricci P, et al. Protection and revascularization of bronchial anastomosis by the intercostal pedicle flap. J Thorac Cardiovasc Surg. 1994;107:1251-4.
2. Allama AM. Intercostal muscle flap for decreasing pain after thoracotomy: a prospective randomized trial. Ann Thorac Surg. 2010;89:195-9.

3. Cerfolio RJ, Bryant AS, Maniscalco LM. A nondivided intercostal muscle flap further reduces pain of thoracotomy: a prospective randomized trial. Ann Thorac Surg. 2008;85:1901-7.

doi:10.1016/j.jtcvs.2010.04.019

\section{TO PUMP, OR NOT TO PUMP, THAT IS THE QUESTION \\ To the Editor:}

We read with great interest the article by Kuss and associates, ${ }^{1}$ who aimed to systematically review all propensity score analyses comparing offand on-pump coronary artery bypass grafting (CABG). Their meta-analysis of 28 studies (100,066 patients) found off-pump CABG superior to on-pump CABG in short-term mortality, the most valid criterion (odds ratio 0.69; $95 \%$ confidence interval $[\mathrm{CI}], 0.60$ 0.75; $P<.0001)$. Meanwhile, Møller and collaborators' meta-analysis ${ }^{2}$ of 57 randomized trials (5202 patients) showed no significant difference in mortality (relative risk [RR] 0.98; 95\% CI, 0.66-1.44). In the future, the largest ongoing randomized trial (CORONARY trial, 4700 patients planned, ClinicalTrials.gov Identifier: NCT00463294) will contribute to the definite answer, as stated by Kuss and colleagues. Shroyer and coworkers ${ }^{3}$ recently reported the results of another large randomized trial (ROOBY trial, 2203 patients enrolled, not included in Møller and associates' meta-analysis). There was no significant difference between off-pump and on-pump CABG in the rate of death from any cause before discharge or within 30 days after the procedure (1. $6 \%$ vs $1.2 \%$; RR $1.38 ; 95 \%$ CI, 0. $68-2.80 ; P=.47)$. Furthermore, even though this result of the ROOBY trial is added to Møller and collaborators' meta-analysis, there is no significant difference in short-term mortality (RR 1.06; 95\% CI, 0.67-1.67; $P=.80$; calculated by us). The evidence from randomized trials obviously demonstrated equivalent short-term mortality 
between off-pump and on-pump CABG, which is contradictory compared with Kuss and colleagues' results from nonrandomized studies.

Late mortality in off-pump CABG is another concern. In the meta-analysis by Wijeysundera and coworkers, ${ }^{4}$ only 2 observational studies reporting risk-adjusted effects on long-term $(\geq 1$ year) outcomes showed essentially no change in mortality (odds ratio $1.01 ; 95 \%$ CI, $0.74-1.40 ; P=.93)$. In the ROOBY trial, ${ }^{3}$ although no significant difference was found for the rate of death from any cause within 1 year (4.1\% vs $2.9 \%$; RR $1.41 ; 95 \%$ CI, $0.90-2.24 ; P=.15)$, the rate of death from cardiac causes within 1 year was higher in the off-pump group than in the on-pump group $(2.7 \%$ vs $1.3 \%$; RR 2.05; 95\% CI, 1.09-3.86; $P=$ $.03)$. Our recent meta-analysis ${ }^{5}$ of 12 randomized trials (4326 patients enrolled) including the ROOBY trial demonstrated a statistically significant increase in late $(\geq 1$ year) all-cause mortality by a factor of 1.37 with offpump relative to on-pump CABG (RR 1.373; 95\% CI, 1.043-1.808; $P=.024)$.

Despite the superiority of off-pump CABG to on-pump CABG in shortterm mortality found in Kuss and associates' meta-analysis ${ }^{1}$ of propensity score analyses, on-pump rather than off-pump CABG should be considered at least for patients who meet the criteria for enrollment in randomized trials (typically for low- to moderate-risk patients) because late, not short-term, mortality reduction must imply the greatest clinical benefit among patients undergoing $\mathrm{CABG}$.

Hisato Takagi, MD, PhD

Takuya Umemoto, MD, PhD

Department of Cardiovascular Surgery

Shizuoka Medical Center Shizuoka, Japan

\section{References}

1. Kuss O, von Salviati B, Börgermann J. Off-pump versus on-pump coronary artery bypass grafting: a systematic review and meta-analysis of propensity score analyses. J Thorac Cardiovasc Surg. 2010 Feb 16 [Epub ahead of print].

2. Møller $\mathrm{CH}$, Penninga L, Wetterslev J, Steinbrüchel DA, Gluud C. Clinical outcomes in randomized trials of off- vs. on-pump coronary artery bypass surgery: systematic review with metaanalyses and trial sequential analyses. Eur Heart $J$. 2008;29:2601-16.

3. Shroyer AL, Grover FL, Hattler B, Collins JF, McDonald GO, Kozora E, et al. On-pump versus off-pump coronary-artery bypass surgery. $N$ Engl J Med. 2009;361:1827-37.

4. Wijeysundera DN, Beattie WS, Djaiani G, Rao V, Borger MA, Karkouti K, et al. Off-pump coronary artery surgery for reducing mortality and morbidity: meta-analysis of randomized and observational studies. J Am Coll Cardiol. 2005;46:872-82.

5. Takagi H, Matusi M, Umemoto T. Off-pump coronary artery bypass may increase late mortality: a meta-analysis of randomized trials. Ann Thorac Surg. 2010;89:1881-8.

doi:10.1016/j.jtcvs.2010.03.044

\section{Reply to the Editor:}

We thank Takagi and Umemoto ${ }^{1}$ for their interest in our recent systematic review of propensity score (PS) analyses in off-pump versus on-pump coronary artery bypass grafting. ${ }^{2}$ As Takagi and Umemoto correctly point out, our estimate for the effect of offpump coronary artery bypass grafting on short-term mortality (odds ratio [OR], 0.69; 95\% confidence interval [CI], 0.60-0.75) differs from the corresponding estimate from randomized controlled trials (RCTs). In their large meta-analysis, Møller and colleagues ${ }^{3}$ found a relative risk of $0.98(95 \% \mathrm{CI}$, 0.66-1.44). Takagi and Umemoto ${ }^{1}$ calculated a relative risk of 1.06 (95\% CI, 0.67-1.67) by adding the results of the ROOBY trial ${ }^{4}$ to Møller and colleagues' results.

Setting aside the subtlety of equating ORs with relative risks, we would like to point out 2 facts. First, even after including the ROOBY results to the data of Møller and colleagues, ${ }^{3}$ the CI for the effect still includes our PS effect estimate of 0.69 . That means that even data from nearly 100 randomized trials on the off-pump/on-pump issue still leave considerable uncertainty (as reflected by the large CI) on the size of the true effect. In particular, ware still far from achieving equivalent short- term mortality, which Takagi and Umemoto $^{1}$ deduce from the current data. This lack of information is also emphasized by Møller and colleagues ${ }^{3}$ in their trial sequential analysis: The authors state that demonstrating equivalence or a minimal clinically relevant effect of the off-pump technique on mortality would require more than 240,000 patients.

Second, and this is also pointed out correctly by Takagi and Umemoto, ${ }^{1}$ we do not expect the effect estimates from PS analyses and RCTs to be equal because the underlying study populations usually differ. Patients in RCTs are, in general, younger and healthier than the average patient. In a study currently under review, we reviewed $28 \mathrm{PS}$ analyses and $51 \mathrm{RCTs}$ that compared off-pump and onpump coronary artery bypass grafting. ${ }^{5}$ We found an average age of 65.8 years and an average left ventricular ejection fraction of $58.8 \%$ in the PS analyses, compared with an average age of 63.1 years and a mean left ventricular ejection fraction of $62.7 \%$ in the RCTs, confirming that patients in the PS analyses are older and in poorer health. After generating similar study populations from PS analyses and RCTs by a meta-matching algorithm (resulting in a meta-matched sample of 10 PS analyses and 29 RCTs), we found an OR for shortterm mortality of $0.53 \quad(95 \% \mathrm{CI}$, $0.43-0.66)$ in the PS analyses and 0.58 (95\% CI, 0.24-1.39) from the RCTs, resulting in an OR difference of -0.05 (95\% CI, -0.56 to 0.47$)$. Unfortunately, this difference is also associated with a large CI, but there is some evidence (supported by other clinical outcome data not shown) that the treatments effects are similar, provided the underlying populations in PS analyses and RCTs are similar.

Finally, we completely agree with Takagi and Umemoto ${ }^{1}$ on the importance of long-term mortality. Sufficient long-term mortality figures were available in 7 PS analyses in our data set, initially accounting for 6813 patients. 